\title{
COMPLETE EXHAUSTIVE SIEVES AND GAMES
}

\author{
RASTISLAV TELGÁRSKY AND HOWARD H. WICKE
}

(Communicated by Dennis Burke)

\begin{abstract}
Properties of completely regular spaces with complete exhaustive sieves are studied using the equivalent notion of partition complete spaces and associated games. Among others the following results are proved. (1) If $X$ is the intersection of countably many partition complete subsets of $Y$, then $X$ is partition complete. (2) If $X$ is $K$-scattered, where $K$ is the class of all partition complete spaces, then $X$ is partition complete. (3) If $X$ is a primitive set in a $C$-scattered space $Y$, then $X$ is the intersection of countably many $C$-scattered subsets of $Y$. (4) The partition completeness is perfect and preserved by open maps.
\end{abstract}

Each space is assumed to be completely regular and $T_{1}$.

DEFINITION 1. A partition $P$ of a space $X$ is called left-open if there is a well-ordering $<$ on $P$ such that $\bigcup\left(P^{\prime}: P^{\prime} \leq P\right)$ is open for every $P \in P$.

Clearly, a space is scattered iff it has a left-open partition into singletons.

DEFINITION 2. A space $X$ is called partition complete if it admits a complete sequence of left-open partitions. A sequence $\left(P_{n}: n<\omega\right)$ of left-open partitions of $X$ is called complete if

(i) $P_{n+1}$ refines $P_{n}$ for all $n<\omega$,

(ii) if $P, P^{\prime} \in P_{n}, Q, Q^{\prime} \in P_{n+1}, Q \subset P, Q^{\prime} \subset P^{\prime}$ and $Q<Q^{\prime}$, then $P \leq P^{\prime}$, and

(iii) if $\left(P_{n}: n<\omega\right)$ is a sequence such that $P_{n+1} \subset P_{n} \in P_{n}$ for all $n<\omega$ and if $\mathcal{F}$ is a filter base in $X$ controlled by $\left(P_{n}: n<\omega\right)$, then $\bigcap\{\bar{F}: F \in \mathcal{F}\} \neq \varnothing$. A filter base $\mathcal{F}$ is controlled by $\left(P_{n}: n<\omega\right)$ if each $P_{n}$ contains some $F \in \mathcal{F}$.

DEFINITION $3[\mathbf{M}]$. A cover $\mathcal{U}$ of a space $X$ is called exhaustive if every nonempty subset $S$ of $X$ has a nonempty relatively open subset of the form $U \cap S$ where $U \in \mathcal{U}$.

The following propositions are easy to check.

Proposition 1. Every left-open partition of $X$ is an exhaustive cover of $X$.

Proposition 2. Every exhaustive cover of $X$ can be refined by a left-open partition of $X$.

DEFINITION $4[\mathbf{M}]$. A sieve $(\mathcal{U}, A, \pi)$ is called complete provided that for every sequence $\left(a_{n}: n<\omega\right)$ with $a_{n} \in A_{n}$ and $\pi_{n}\left(a_{n+1}\right)=a_{n}$, if $\mathcal{F}$ is a filter base controlled by $\left(U_{a_{n}}: n<\omega\right)$, then $\bigcap\{\bar{F}: F \in \mathcal{F}\} \neq \varnothing$. A sieve is called exhaustive if $\left\{U_{b}: b \in \pi_{n}^{-1}(a)\right\}$ is an exhaustive cover of $U_{a}$ for every $a \in A_{n}$ and $n<\omega$.

Received by the editors June 26, 1986 and, in revised form, November 4, 1986.

1980 Mathematics Subject Classification (1985 Revision). Primary 54D30, 54E18; Secondary $90 \mathrm{D} 42$.

Key words and phrases. Partition complete space, sieve complete space, $K$-scattered space, exhaustive sieve, open presieve, primitive set, perfect mapping, open mapping, topological game. 
The following theorem can be seen to be equivalent to Proposition 4.1(b) and (d) of $[\mathbf{M}]$, which involves sieves with pairwise disjoint levels.

THEOREM 1 [M]. A space $X$ has a complete exhaustive sieve iff $X$ is partition complete.

The next two theorems provide evidence for significance of complete exhaustive sieves.

THEOREM $2[\mathbf{M}]$. A metrizable space is completely metrizable iff it has a complete exhaustive sieve.

THEOREM $3[\mathrm{~W}]$. A paracompact space is Čech complete iff it is a p-space having a complete exhaustive sieve. A space has a complete open sieve (i.e., it is sieve-complete) iff it has a complete exhaustive sieve and is a monotonic $p$-space.

Michael's game $M(X)$. Players I and II alternately choose nonempty subsets $S_{0} \supset T_{0} \supset S_{1} \supset T_{1} \supset \cdots$ of $X$ such that $T_{n}$ is relatively open in $S_{n}$ for all $n<\omega$. Player II wins iff whenever $\mathcal{F}$ is a filter base in $X$ controlled by $\left(T_{n}: n<\omega\right)$, then $\bigcap\{\bar{F}: F \in \mathcal{F}\} \neq \varnothing$.

THEOREM $4[\mathbf{M}]$. For a regular space $X$, the following are equivalent:

(a) $X$ has a complete exhaustive sieve;

(b) Player II has a stationary winning strategy for $M(X)$;

(c) Player II has a winning strategy for $M(X)$.

Although all proofs given below can be presented in the framework of Definition 2 , it turns out to be more convenient and transparent to use the game-theoretic characterization of partition completeness. We shall use Theorems 1 and 4 in the subsequent proofs without special mention.

THEOREM 5. If $X_{n} \subset Y$ each $X_{n}$ is partition complete, then $X=\bigcap\left\{X_{n}: n<\right.$ $\omega\}$ is partition complete.

PROOF. Without loss of generality we may assume that $X \neq \varnothing$. Let $\sigma_{n}$ be a stationary winning strategy of Player II in $M\left(X_{n}\right)$ for all $n<\omega$. We shall define a winning strategy $\sigma$ for Player II in $M(X)$. Let $\left\{N_{k}: k<\omega\right\}$ be a partition of $\omega$ into infinite sets. Given any $n$ and a partial play $\left(S_{0}, T_{0}, \ldots, S_{n}\right)$ of $M(X)$, we define $\sigma\left(S_{0}, \ldots, S_{n}\right)=\sigma_{k}\left(S_{n}\right)$, where $k$ is the unique number such that $n \in N_{k}$. Let $\left(S_{0}, T_{0}, S_{1}, T_{1}, \ldots\right)$ be a play consistent with $\sigma$. Let $₹$ be a filter base (of subsets of $X)$ controlled by $\left(T_{n}: n<\omega\right)$. Without loss of generality we may assume that $\mathcal{F}$ has at most one cluster point in $Y$ (for instance, let $y \in \bigcap\{\bar{F}: F \in \mathcal{F}\}$, where the closure is taken in $\beta X$, and let $U$ be a neighborhood base at $y$ in $\beta X$; then $\{F \cap U: F \in \mathcal{F} \& U \in U\}$ is a filter base in $X$, finer than $\mathcal{F}$, and having at most one cluster point in $X)$. We shall show that $\bigcap\{\bar{F} \cap X: F \in \mathcal{F}\} \neq \varnothing$. Fix a $k<\omega$ and write $N_{k}$ as $\{k(n): n<\omega\}$, where $k(0)<k(1)<\cdots$. Then $T_{k(n)}=\sigma_{k}\left(S_{k(n)}\right)$ for each $n<\omega$, i.e., $\left(S_{k(0)}, T_{k(0)}, S_{k(1)}, T_{k(1)}, \ldots\right)$ is a play of $M\left(X_{k}\right)$ consistent with $\sigma_{k}$. Therefore $\bigcap\left\{\bar{F} \cap X_{k}: F \in \mathcal{F}\right\}=\left\{x_{k}\right\}$ for some $x_{k} \in X_{k}$. Since $\mathcal{F}$ has at most one cluster point in $Y$ it follows that $x_{0}=x_{1}=\cdots$. Hence $\bigcap\{\bar{F} \cap X: F \in \mathcal{F}\}=\left\{x_{0}\right\}$. 
THEOREM 6. If $X_{n}$ is partition complete for all $n<\omega$, then $\mathbf{P}\left\{X_{n}: n<\omega\right\}$ is partition complete.

PrOOF. Observe that if $X$ is partition complete and $Y$ is compact, then $X \times Y$ is partition complete. Let $\left(X_{n}: n<\omega\right)$ be a sequence of partition complete spaces, $Y$ be a compact space containing all $X_{n}$ 's, and $Y_{n}=\left\{y \in Y^{\omega}: y(n) \in X_{n}\right\}$. Then each $Y_{n}$ is partition complete and therefore $\bigcap\left\{Y_{n}: n<\omega\right\}$ is partition complete by Theorem 5 . Since the last set is homeomorphic to $\mathbf{P}\left\{X_{n}: n<\omega\right\}$, the theorem follows.

DEFINITION $5\left[\mathbf{T}_{\mathbf{2}}\right]$. A space $X$ is called $K$-scattered, where $K$ is a class of spaces, if for each nonempty closed subset $E$ of $X$ there is an open set $U$ in $X$ such that $E \cap U \neq \varnothing$ and $\overline{E \cap U} \in K$.

PROPOSITION 3 [JST]. If $K$ is a class of spaces such that $E \in K$ whenever $E$ is a closed subspace of a space in $K$, then the following are equivalent:

(a) $X$ is $K$-scattered;

(b) $X$ has a left-open partition $P$ such that $\bar{P} \in K$ for all $P \in P$.

THEOREM 7. If $X$ is a $K$-scattered space, where $K$ is the class of partition complete spaces, then $X$ is partition complete.

Proof. By Proposition 3, $X$ has a left-open partition $P$ such that $\bar{P} \in K$ for all $P \in P$. Therefore Player II has a stationary winning strategy $\sigma_{P}$ in $M(\bar{P})$. We define a strategy $\sigma$ for Player II to win $M(X)$ as follows. Suppose $S_{0}$ is the first move of Player I in a play of $M(X)$. Let $P$ be the first member of $P$ such that $P \cap S_{0} \neq \varnothing$. Put $\sigma\left(S_{0}\right)=\sigma_{P}\left(S_{0} \cap P\right)$. Since $S_{0} \cap P$ is relatively open in $S_{0}$, the set $\sigma\left(S_{0}\right)$ is relatively open in $S_{0}$. Let $T_{0}=\sigma\left(S_{0}\right)$ and let $S_{1} \subset T_{0}$. Then we put $\sigma\left(S_{0}, S_{1}\right)=\sigma_{P}\left(S_{1}\right)$, etc. Clearly, the play continues in $P$. If $\left(S_{0}, T_{0}, S_{1}, T_{1}, \ldots\right)$ is a play in $M(X)$, then if $P$ is as above, $T_{n} \subset P$ for all $n<\omega$. Moreover, $\left(S_{0} \cap P, T_{0}, S_{1}, T_{1}, \ldots\right)$ is a play in $M(\bar{P})$ consistent with $\sigma_{P}$. If $\mathcal{F}$ is a filter base controlled by $\left(T_{n}: n<\omega\right)$, then so is $\{F \cap P: F \in \mathcal{F}\}$. Hence

$$
\varnothing \neq \bigcap\{\overline{F \cap P}: F \in \mathcal{F}\} \subset \bigcap\{\bar{F}: F \in \mathcal{F}\} \subset \bar{P},
$$

so $X$ is partition complete.

Theorem 7 does not hold for sieve completeness (see Example 2 below).

It is easy to check that closed subsets and open subsets of a partition complete space are partition complete. From Theorem 7 we get a stronger result.

COROLLARY 1. If $X$ is a $K$-scattered subset of a partition complete space $Y$, where $K$ is the collection of all closed subsets of $Y$, then $X$ is partition complete. In other words: If $X$ is a resolvable subset (see [ $\left.\mathbf{T}_{\mathbf{1}}\right]$ ) of a partition complete space $Y$, then $X$ is partition complete.

Let $C$ and $\check{C}$ denote the class of compact spaces and Cech complete spaces

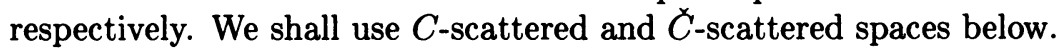

COROLlaRY 2. If $\left(X_{n}: n<\omega\right)$ is a sequence of $C$-scattered (or $\check{C}$-scattered) subsets of a space $Y$, then $X=\bigcap\left\{X_{n}: n<\omega\right\}$ is partition complete.

The next example shows that a space $X$ need not be $C$-scattered if it is the

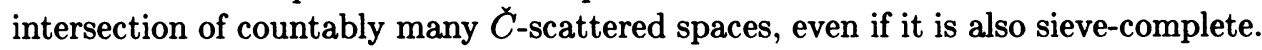


EXAMPLE 1. A sieve complete space $X$ that is nowhere locally Cech complete (thus is not $\check{C}_{\text {-scattered). }}$

CONSTRUCTION. Let $X=Y^{\omega}$, where

$$
Y=\left(\omega_{1}+1\right)^{2} \backslash\left(\left\{\left(\omega_{1}, \alpha+1\right): \alpha<\omega_{1}\right\} \cup\left\{\left(\omega_{1}, \omega_{1}\right)\right\}\right) .
$$

The space $Y$ is sieve complete, because $\left(\omega_{1}+1\right)^{2} \backslash Y$ is compact-like (see [ $\left.\mathbf{T}_{\mathbf{3}}\right]$ ). Furthermore, $Y$ is not Cech complete, because $\left(\omega_{1}+1\right)^{2} \backslash Y$ is not $\sigma$-compact. Finally, $X$ is nowhere locally Cech complete, because $Y$ is not Cech complete.

The notion of a presieve was introduced in $[\mathbf{W}]$, while the (equivalent) original definition was given in terms of primitive sequences (see $\left[\mathbf{W} \mathbf{W}_{\mathbf{1}}, \mathbf{W} \mathbf{W}_{\mathbf{2}}\right]$ ).

DEFINITION 6. Let $X \subset Y$. A presieve of $X$ in $Y$ is a triple $(\mathcal{U}, A, \pi)=$ $\left(\left(\mathcal{U}_{n}, A_{n}, \pi_{n}\right): n<\omega\right)$ such that for each $n<\omega$ :

(a) $A_{n}$ is well ordered;

(b) $\pi_{n}: A_{n+1} \rightarrow A_{n}$ is order preserving, i.e., if $a<b$ then $\pi_{n}(a) \leq \pi_{n}(b)$;

(c) $U_{n}=\left\{U_{a}: a \in A_{n}\right\}$ is a family of subsets of $Y$;

(d) $X \subset \bigcup\left\{U_{a}: a \in A_{n}\right\}$;

(e) $U_{a} \subset U_{\pi_{n}(a)}$ for every $a \in A_{n+1}$;

(f) $X \cap p\left(U_{a}, \mathcal{U}_{n+1}\right) \subset p\left(U_{\pi_{n}(a)}, \mathcal{U}_{n}\right)$ for every $a \in A_{n+1}$, where $p\left(U_{a}, \mathcal{U}_{n}\right)=$ $U_{a} \backslash \bigcup\left\{U_{b}: b<a\right\} ; p\left(U_{a}, U_{n}\right)$ is called the primitive part of $U_{a}$.

DEFINITION $7[\mathbf{P h}]$. A subset $X$ of a space $Y$ is a primitive set in $Y$ if there is an open (in $Y$ ) presieve $(\mathcal{U}, A, \pi)$ of $X$ in $Y$ such that

$$
\bigcap\left\{\left(U_{a_{n}}, U_{n}\right): n<\omega\right\} \subset X
$$

for every sequence $\left(a_{n}: n<\omega\right)$ such that $\pi_{n}\left(a_{n+1}\right)=a_{n}$ whenever $n<\omega$.

Note that the following kinds of sets are primitive sets: open sets, $G_{\delta}$ sets, sets of interior condensation (i.e., $W_{\delta}$ sets), and primitive sets of interior condensation [W $\mathbf{W}_{\mathbf{2}}$ ] (i.e., subsets $X$ having an open presieve $(\mathcal{U}, A, \pi)$ such that $\bigcap\left\{U_{a_{n}}: n<\right.$ $\omega\} \subset X$ whenever $\bigcap\left\{p\left(U_{a_{n}}, U_{n}\right): n<\omega\right\} \neq \varnothing$ where $\pi_{n}\left(a_{n+1}\right)=a_{n}$ for every $n<\omega)$.

THEOREM 8. If $X$ is a primitive set in a partition complete space $Y$, then $X$ is partition complete.

ProOF. Let $(U, A, \pi)$ be an open presieve for $X$ in $Y$ such that $\bigcap\left\{p\left(U_{a_{n}}, \mathcal{U}_{n}\right): n\right.$ $<\omega\} \subset X$ whenever $\pi_{n}\left(a_{n+1}\right)=a_{n}$ for every $n<\omega$, and let $\sigma$ be a winning strategy of Player II in $M(Y)$. We shall define a winning strategy $\tau$ for Player II in $M(X)$ as follows. If $S_{n}$ is a nonempty subset of $X$ chosen at move $n$, we find the first $a_{n}$ in $A_{n}$ such that $S_{n} \cap U_{a_{n}} \neq \varnothing$. Clearly, the set $S_{n} \cap U_{a_{n}}$ is relatively open in $S_{n}$. Let $V_{n}$ be a nonempty open set in $Y$ such that $V_{n} \cap S_{n} \neq \varnothing$ and $\bar{V}_{n} \subset U_{a_{n}}$. We put $\tau\left(S_{n}\right)=\sigma\left(S_{n} \cap V_{n}\right)$. It is easy to verify that $\tau$ is a winning strategy.

EXAMPLE 2. A partition complete (scattered Lindelöf) space $X$ that is not a primitive set in $\beta X$ (hence $X$ is not sieve-complete).

CONSTRUCTION. Let $X=\omega \cup\{p\}$ be a subspace of $\beta \omega$, where $p \in \beta \omega \backslash \omega$. Clearly, $X$ is a scattered Lindelöf space. Suppose $(U, A, \pi)$ is an open presieve of $X$ in $\beta \omega$. Let $a_{n}=\min \left\{a \in A_{n}: p \in U_{a}\right\}$. Then $p\left(U_{a_{n}}, U_{n}\right)=U_{a_{n}} \backslash V_{n}$ for some $V_{n} \subset \omega$. Let $V=\bigcup\left(V_{n}: n<\omega\right)$. Then $W=\bigcap\left\{p\left(U_{a_{n}}, U_{n}\right): n<\omega\right\}=\bigcap\left\{U_{a_{n}}: n<\omega\right\} \backslash V$. Since $W$ is a $G_{\delta}$ set containing the point $p$, it must be uncountable. Hence $W$ is not a subset of $X$, and therefore $X$ is not a primitive set in $\beta \omega$. 
Note that if $X$ is sieve-complete and if $Y$ is a compactification of $X$, then $X$ is a primitive set in $Y$. In $\left[\mathbf{T}_{\mathbf{3}}\right]$ it was shown that each sieve-complete space is the intersection of countably many $C$-scattered spaces. In Theorem 9 we get a more general result.

THEOREM 9. If $X$ is a primitive set in a $C$-scattered space $Y$, then $X$ is the intersection of countably many $C$-scattered subsets of $Y$.

ProOF. Let $S_{n}$ be the set of all $\left(a_{0}, \ldots, a_{n}\right) \in A_{0} \times A_{1} \times \cdots<A_{n}$ such that

$$
\pi_{0}\left(a_{1}\right)=a_{0}, \pi_{1}\left(a_{2}\right)=a_{1}, \ldots, \pi_{n-1}\left(a_{n}\right)=a_{n-1} .
$$

Let

$$
P\left(a_{0}, \ldots, a_{n}\right)=\bigcap\left\{p\left(U_{a_{k}}, \mathcal{U}_{k}\right): 0 \leq k \leq n\right\}
$$

and

$$
X_{n}=\bigcup\left\{P\left(a_{0}, \ldots, a_{n}\right):\left(a_{0}, \ldots, a_{n}\right) \in S_{n}\right\} .
$$

Claim 1. $\bigcap\left\{X_{n}: n<\omega\right\}=X$.

Let $x \in X$. Then there is a unique sequence $\left(a_{0}, a_{1}, \ldots\right)$ such that $\left(a_{0}, \ldots, a_{n}\right) \in$ $S_{n}$ for every $n<\omega$ and $x \in \bigcap\left\{p\left(U_{a_{n}}, \mathcal{U}_{n}: n<\omega\right\}\right.$. Therefore $x \in P\left(a_{0}, \ldots, a_{n}\right) \subset$ $X_{n}$ for every $n<\omega$. To show the converse inclusion, assume that $x \in \bigcap\left\{X_{n}: n<\right.$ $\omega\}$. Then there is a unique $a_{0} \in A_{0}$ such that $x \in p\left(U_{a_{0}}, U_{0}\right)$, there is a unique $a_{1} \in A_{1}$ such that $\left(a_{0}, a_{1}\right) \in S_{1}$ and $x \in p\left(U_{a_{1}}, \mathcal{U}_{1}\right)$, and so on. Hence $x \in$ $\bigcap\left\{p\left(U_{a_{n}}, U_{n}\right): n<\omega\right\} \subset X$.

ClaIM 2. Each $X_{n}$ is $C$-scattered.

Since $X_{0}$ is open in $Y$, it is $C$-scattered. Moreover, it is easy to see that each $p\left(U_{a}, U_{n}\right)$ is $C$-scattered, and therefore each $P\left(a_{0}, \ldots, a_{n}\right)$ is also $C$-scattered. Let $F$ be a nonempty relatively closed subset of $X_{n+1}$. Find the first $a_{0} \in A_{0}$ such that $F \cap p\left(U_{a_{0}}, \mathcal{U}_{0}\right) \neq \varnothing$, find the first $a_{1} \in A_{1}$ such that $\left(a_{0}, a_{1}\right) \in S_{1}$ and $F \cap p\left(U_{a_{1}}, \mathcal{U}_{1}\right) \neq \varnothing$, and so on, until $\left(a_{0}, a_{1}, \ldots, a_{n}, a_{n+1}\right)$ is defined. Now $F \cap$ $P\left(a_{0}, a_{1}, \ldots, a_{n}, a_{n+1}\right)$ is nonempty, $C$-scattered, and relatively open in $F$. Thus $F$ has a point of local compactness.

Actually, a more general statement than Theorem 9 is valid (and can be proved in the same way): If $X$ is a primitive set in $Y$, then $X$ is the intersection of countably many $K$-scattered subsets of $Y$, where $K$ is the collection of all closed subsets of $Y$.

Question 1. Does a converse to Theorem 9 hold?

From Theorem 9 and Corollary 2 we get

COROLlaRY 3. If $X$ is a primitive set in a $C$-scattered space $Y$, then $X$ is partition complete.

However, Corollary 3 can also be derived from Theorem 7 and Theorem 8 .

EXAMPLE 3. A dense subset $X$ of a compact space $Y$ such that $X$ is a primitive set in $Y$, but $X$ is not a $W_{\delta}$-set in $Y$ (that is, $X$ is not sieve-complete).

Construction. Let $Y$ be the long line with endpoints. Points of $Y$, except for the last point $\omega_{1}$, can be uniquely represented as $\alpha+r$, where $\alpha \in\left[0, \omega_{1}\right)$ and $r \in[0,1)$. Let $X=Y \backslash\left\{\alpha+1: \alpha<\omega_{1}\right\}$ and

$$
\nvdash_{n}=\{[0,1)\} \cup\left\{(\alpha+1, \alpha+2): \alpha<\omega_{1}\right\} \cup\left\{\left(\alpha_{n}(\lambda), \lambda+1\right): \lambda \in \Lambda\right\} \cup\{X\},
$$


where $\Lambda$ is the set of all countable limit ordinals and $\left\{\alpha_{n}(\lambda): n<\omega\right\}$ is a fixed increasing sequence of ordinals with $\lim \alpha_{n}(\lambda)=\lambda$. Note that $X$ is a $C$-scattered Lindelöf space and $X$ is dense in $Y$. However, $Y \backslash X=\left\{\alpha+1: \alpha<\omega_{1}\right\}$ is an uncountable discrete space, so it is not Lindelöf. Hence $X$ is not sieve-complete by Theorem 1 of $\left[\mathbf{T}_{3}\right]$. Finally, $\left(\mathcal{H}_{n}: n<\omega\right)$, where every $\mathcal{H}_{n}$ is ordered in the natural way as written above, determines a sequence $\left(\left(U_{n}, A_{n}, \pi_{n}\right): n<\omega\right)$ satisfying the conditions of Definitions 6 and 7. Thus $X$ is a primitive set in $Y$.

Question 2. Is there a first countable space $Y$ with a dense subset $X$ satisfying the conditions of Example 3? Is there a first countable partition complete space $X$ that is not sieve-complete? (Such a space $X$ cannot be scattered.)

THEOREM 10. Let $X$ be a partition complete space and $f$ be a perfect mapping from $X$ onto $Y$. Then $Y$ is partition complete.

ProOF. Let $\sigma$ be a winning strategy of Player II in $M(X)$. We shall define a winning strategy $\tau$ for Player II in $M(Y)$.

ClAIM. If $S$ is a nonempty subset of $Y$ and if $E$ is a relatively closed nonempty subset of $f^{-1}(S)$, then there is a relatively closed subset $\mu(E)$ of $E$ such that $f(\mu(E))=f(E)$ and $f\left(E^{\prime}\right) \neq f(E)$ for every relatively closed proper subset $E^{\prime}$ of $\mu(E)$.

The proof of the claim is standard and therefore is omitted.

Let $S_{0}$ be a nonempty subset of $Y$. Put $S_{0}^{\prime}=\mu\left(f^{-1}\left(S_{0}\right)\right), T_{0}^{\prime}=\sigma\left(S_{0}^{\prime}\right), T_{0}=$ $S_{0} \backslash f\left(S_{0}^{\prime} \backslash T_{0}^{\prime}\right)$, and $\tau\left(S_{0}\right)=T_{0}$. Observe that $T_{0}$ is relatively open in $S_{0}$. Moreover, $T_{0} \neq \varnothing$. For, if $T_{0} \neq \varnothing$, then $f\left(S_{0}^{\prime} \backslash T_{0}^{\prime}\right)=S_{0}$; since $f\left(S_{0}^{\prime}\right)=S_{0}$, we have $S_{0}^{\prime} \backslash T_{0}^{\prime}=S_{0}^{\prime}$, that is $T_{0}^{\prime}=\varnothing$, a contradiction. Let $S_{1}$ be a nonempty subset of $T_{0}$. Then $S_{1} \cap f\left(S_{0}^{\prime} \backslash T_{0}^{\prime}\right)=\varnothing$, hence $f^{-1}\left(S_{1}\right) \cap\left(S_{0}^{\prime} \backslash T_{0}^{\prime}\right)=\varnothing$, and consequently $f^{-1}\left(S_{1}\right) \cap S_{0}^{\prime} \subset T_{0}^{\prime}$. Since $f^{-1}\left(S_{1}\right) \cap S_{0}^{\prime}$ is relatively closed in $f^{-1}\left(S_{1}\right)$, we put $S_{1}^{\prime}=$ $\mu\left(f^{-1}\left(S_{1}\right) \cap S_{0}^{\prime}\right)$. Observe that $S_{1}^{\prime} \neq \varnothing$. Put $T_{1}^{\prime}=\sigma\left(S_{1}^{\prime}\right), T_{1}=S_{1} \backslash f\left(S_{1}^{\prime} \backslash T_{1}^{\prime}\right)$, and $\tau\left(S_{0}, S_{1}\right)=T_{1}$. Observe that $T_{1}$ is relatively open in $S_{1}$. Moreover, $T_{1} \neq \varnothing$. For, if $T_{1}=\varnothing$, then $S_{1} \subset f\left(S_{1}^{\prime} \backslash T_{1}^{\prime}\right) \subset f\left(S_{1}^{\prime}\right)=S_{1}$, and hence $S_{1}^{\prime}=S_{1}^{\prime} \backslash T_{1}^{\prime}$, that is $T_{1}^{\prime}=\varnothing$, a contradiction. Continuing in this manner we get a play $\left(S_{0}^{\prime}, T_{0}^{\prime}, S_{1}^{\prime}, T_{1}^{\prime}, \ldots\right)$ of $M(X)$ consistent with $\sigma$ and a play $\left(S_{0}, T_{0}, S_{1}, T_{1}, \ldots\right)$ of $M(Y)$. Let $\mathcal{F}$ be a filter base in $Y$ controlled by $\left(T_{n}: n<\omega\right)$. Then $\left\{f^{-1}(F) \cap S_{n}^{\prime}: F \in \mathcal{F} \& n<\omega\right\}$ is a filter base in $X$ controlled by $\left(T_{n}^{\prime}: n<\omega\right)$. For, assume that $F \subset T_{n}$ for some $F \in \mathcal{F}$ and some $n<\omega$. Then $F \subset S_{n} \backslash f\left(S_{n}^{\prime} \backslash T_{n}^{\prime}\right)$, so $F \cap f\left(S_{n}^{\prime} \backslash T_{n}^{\prime}\right)=\varnothing$. Hence $f^{-1}(F) \cap\left(S_{n}^{\prime} \backslash T_{n}^{\prime}\right)=\varnothing$, and consequently $f^{-1}(F) \cap S_{n}^{\prime} \subset T_{n}^{\prime}$. Now we show that $f^{-1}(F) \cap S_{n}^{\prime} \neq \varnothing$ for every $F \in \mathcal{F}$ and every $n<\omega$. Suppose to the contrary that $f^{-1}(F) \cap S_{n}^{\prime}=\varnothing$ for some $F \in \mathcal{F}$ and some $n<\omega$. If $n=0$, then $f\left(S_{0}^{\prime}\right)=S_{0}$, so we get $F \cap S_{0}=\varnothing$, a contradiction. If $n>0$, then $S_{n}^{\prime}=\mu\left(f^{-1}\left(S_{n}\right) \cap S_{n-1}^{\prime}\right)$ and $f\left(S_{n}^{\prime}\right)=f\left(f^{-1}\left(S_{n}\right) \cap S_{n-1}^{\prime}\right)=S_{n}$, thus $F \cap S_{n}=\varnothing$, a contradiction. Since $\sigma$ is a winning strategy, we have

$$
\bigcap\left\{\overline{f^{-1}(F) \cap T_{n}^{\prime}}: F \in \mathcal{F} \& n<\omega\right\} \neq \varnothing .
$$

Now, since

$$
\overline{f^{-1}(F) \cap T_{n}^{\prime}} \subset \overline{f^{-1}(F)} \subset f^{-1}(\bar{F})
$$

and

$$
\bigcap\left\{f^{-1}(\bar{F}): F \backslash \mathcal{F}\right\}=f^{-1}(\bigcap\{\bar{F}: F \in \mathcal{F}\}),
$$

it follows that $\bigcap\{\bar{F}: F \in \mathcal{F}\} \neq \varnothing$. 
The following example shows that a closed continuous image of a partition complete space need not be partition complete.

EXAMPLE 4. A partition complete space $X$ and a closed continuous mapping $f$ from $X$ onto a first category space.

Construction. Actually, a variant of this example is used in [Pu, Example 2] for another purpose. Let $X=\bigoplus\left\{B^{n}: 0<n<\omega\right\}$, where $B=\{\alpha+1: \alpha<$ $\left.\omega_{1}\right\} \cup\left\{\omega_{1}\right\}$. Then $X$ is a Lindelöf scattered $P$-space. Let $Y=\left\{y \in\left(B^{\omega}\right)_{\delta}: \exists n<\omega\right.$ $\left.\forall k>n y(k)=\omega_{1}\right\}$, where $\left(B^{\omega}\right)_{\delta}$ denotes the space $B^{\omega}$ endowed with the $G_{\delta^{-}}$ topology. Then $Y$ is a Lindelöf self-dense $P$-space. Finally, let $x \in B^{n}$; we put $f(x)(k)=x(k)$ if $k \leq n$ and $f(x)(k)=\omega_{1}$ if $k>n$. Clearly, each $f\left(B^{n}\right)$ is nowhere dense in $f\left(B^{n+1}\right)$, thus $Y$ is of the first category. Since $X$ and $Y$ are Lindelöf $P$-spaces, their Lindelöf subsets and closed subsets coincide. Since $f$ is continuous, the image of a Lindelöf set in $X$ is a Lindelöf set in $Y$. Therefore the mapping $f$ is closed.

THEOREM 11. If $f$ is a perfect mapping from a space $X$ onto a partition complete space $Y$, then $X$ is partition complete.

PROOF. Let $\sigma$ be a winning strategy for Player II in $M(Y)$. We define a winning strategy $\tau$ for Player II in $M(X)$ as follows. Given a nonempty subset $S$ of $X$, we put

$$
\tau(S)=S \cap f^{-1}(\sigma(f(S))) .
$$

Since $f^{-1}(\sigma(f(S)))$ is relatively open in $f^{-1}(f(S))$ and $S \subset f^{-1}(f(S))$, it follows that $\tau(S)$ is relatively open in $S$. Moreover, $\tau(S) \neq \varnothing$. For, suppose that $\tau(S)=\varnothing$. Then $f(S) \cap \sigma(f(S))=\varnothing$, so $\sigma(f(S))=\varnothing$, a contradiction. Let $\left(S_{0}, T_{0}, S_{1}, T_{1}, \ldots\right)$ be a play of $M(X)$ consistent with $\tau$ and $₹$ be a filter base in $X$ controlled by $\left(T_{n}: n<\omega\right)$. Put $S_{n}^{\prime}=f\left(S_{n}\right)$ and $T_{n}^{\prime}=\sigma\left(S_{n}^{\prime}\right)$. Then $T_{n}=S_{n} \cap f^{-1}\left(T_{n}^{\prime}\right)$. Clearly, $\{f(F): F \in \mathcal{F}\}$ is a filter base in $Y$. Suppose that $F \in \mathcal{F}$ and $F \subset T_{n}=$ $S_{n} \cap f^{-1}\left(T_{n}^{\prime}\right)$ for some $n<\omega$. Since $F \subset f^{-1}\left(T_{n}^{\prime}\right)$, we have $f(F) \subset f\left(f^{-1}\left(T_{n}^{\prime}\right)\right)=$ $T_{n}^{\prime}$. Therefore $\{f(F): F \in \mathcal{F}\}$ is controlled by $\left(T_{n}^{\prime}: n<\omega\right)$ and consequently $\bigcap\{\overline{f(F)}: F \in \mathcal{F}\} \neq \varnothing$. Pick a point $y$ in $\bigcap\{\overline{f(F)}: F \in \mathcal{F}\}$ and fix $F \in \mathcal{F}$. Since $\overline{f(F)} \subset f(\bar{F})$, we have $y \in f(\bar{F})$, and consequently $f^{-1}(y) \cap \bar{F} \neq \varnothing$. Since $f^{-1}(y)$ is compact, $\bigcap\left\{f^{-1}(y) \cap \bar{F}: F \in \mathcal{F}\right\} \neq \varnothing$. Therefore, $\bigcap\{\bar{F}: F \in \mathcal{F}\} \neq \varnothing$.

For the proof of the next theorem it is convenient to use the following modification of the game $M(X)$. Players I and II choose nonempty subsets $S_{0}, W_{0}, S_{1}, W_{1}, \ldots$ of $X$ so that for each $n<\omega: S_{n+1} \subset W_{n} \cap S_{n}, W_{n+1} \subset W_{n}$, and $W_{n}$ is open in $X$. Player II wins the play $\left(S_{0}, W_{0}, S_{1}, W_{1}, \ldots\right)$ iff whenever $₹$ is a filter base in $X$ controlled by $\left(W_{n} \cap S_{n}: n<\omega\right)$, then $\bigcap\{\bar{F}: F \in \mathcal{F}\} \neq \varnothing$. Denote this game by $M^{\prime}(X)$. It is easy to show that Player II has a winning strategy in $M^{\prime}(X)$ iff Player II has a winning strategy in $M(X)$.

THEOREM 12. If $X$ is partition complete, and if $Y$ is an open continuous image of $X$, then $Y$ is partition complete.

Proof. Let $f$ be an open continuous map from $X$ onto $Y$ and $\sigma$ be a winning strategy of Player II in $M^{\prime}(X)$. We shall define a winning strategy $\tau$ for Player II in $M^{\prime}(Y)$. Let $S_{0}$ be a nonempty set in $Y$. Put $S_{0}^{\prime}=f^{-1}\left(S_{0}\right), W_{0}^{\prime}=\sigma\left(S_{0}^{\prime}\right)$, $W_{0}=f\left(W_{0}^{\prime}\right)$ and $\tau\left(S_{0}\right)=W_{0}$. Let $S_{1}$ be a nonempty subset of $W_{0} \cap S_{0}$. Put $S_{1}^{\prime}=W_{0}^{\prime} \cap f^{-1}\left(S_{1}\right), W_{1}^{\prime}=\sigma\left(S_{0}^{\prime}, W_{0}^{\prime}, S_{1}^{\prime}\right), W_{1}=f\left(W_{1}^{\prime}\right)$, and $\tau\left(S_{0}, W_{0}, S_{1}\right)=W_{1}$. 
Continuing in this manner we get a play $\left(S_{0}^{\prime}, W_{0}^{\prime}, S_{1}^{\prime}, W_{1}^{\prime}, \ldots\right)$ of $M^{\prime}(X)$ consistent with $\sigma$ and a play $\left(S_{0}, W_{0}, S_{1}, W_{1}, \ldots\right)$ of $M^{\prime}(Y)$. Let $₹$ be a filter base in $Y$ controlled by $\left(S_{n} \cap W_{n}: n<\omega\right)$. Then

$$
\mathcal{F}^{\prime}=\left\{f^{-1}(F) \cap S_{n}^{\prime} \cap W_{n}^{\prime}: F \in \mathcal{F} \& n<\omega\right\}
$$

is a filter base in $X$ controlled by $\left(S_{n}^{\prime} \cap W_{n}^{\prime}: n<\omega\right)$. Hence $\bigcap\left\{\bar{F}^{\prime}: F^{\prime} \in \mathcal{F}^{\prime}\right\} \neq \varnothing$. Since

$$
\begin{aligned}
f\left(\bigcap\left\{\overline{F^{\prime}}: F^{\prime} \in \mathcal{F}^{\prime}\right\}\right) & \subset \bigcap\left\{f\left(\overline{F^{\prime}}\right): F^{\prime} \in \mathcal{F}^{\prime}\right\} \subset \bigcap\left\{\overline{f\left(F^{\prime}\right)}: F^{\prime} \in \mathcal{F}^{\prime}\right\} \\
& \subset \bigcap\{\bar{F}: F \in \mathcal{F}\},
\end{aligned}
$$

we have $\bigcap(\bar{F}: F \in \mathcal{F}) \neq \varnothing$. Therefore $Y$ is partition complete.

We note that a brief proof of Theorem 12 can be given by observing that an open continuous image of a complete exhaustive sieve is a complete exhaustive sieve.

Concluding, we summarize the main completeness properties considered in this paper.

COROLlaRY 4. (a) $\Rightarrow$ (b) $\Rightarrow$ (c) $\Rightarrow$ (d) $\Rightarrow$ (e) $\Rightarrow$ (f) $\Rightarrow$ (g), where

(a) $X$ is a $G_{\delta}$ set in $\beta X$, i.e., $X$ is Čech complete;

(b) $X$ is a $W_{\delta}$ set in $\beta X$, i.e., $X$ is sieve-complete;

(c) $X$ is a primitive set in $\beta X$;

(d) $X$ is a primitive set in a $C$-scattered space;

(e) $X$ is the intersection of countably many $C$-scattered sets;

(f) $X$ is the intersection of countably many $\check{C}$-scattered sets;

(g) $X$ is partition complete, i.e., $X$ has a complete exhaustive sieve.

\section{REFERENCES}

[JST] H. J. K. Junnila, J. C. Smith and R. Telgársky, Closure-preserving covers by small sets, Topology and Appl. 23 (1986), 237-262.

[M] E. Michael, A note on completely metrizable spaces, Proc. Amer. Math. Soc. 96 (1986), 512522.

[Ph] T. M. Phillips, Extending continuous functions, Topology Proc. (Memphis State Univ. Conf.) Dekker, New York, 1976, pp. 229-237.

[Pu] S. Purish, The orderability and closed images of scattered spaces, preprint, 1986.

[T $\left.\mathbf{T}_{1}\right] \mathrm{R}$. Telgársky, $C$-scattered and paracompact spaces, Fund. Math. 73 (1971), 59-74.

$\left[\mathbf{T}_{\mathbf{2}}\right]$ - Space defined by topological games, Fund. Math. 88 (1975), 193-223.

[T $\left.\mathbf{T}_{\mathbf{3}}\right]$, On sieve-complete and compact-like spaces, Topology and Appl. 16 (1983), 61-68.

[W] H. H. Wicke, Complete exhaustive sieves, Topology Proc. (to appear).

[WW $]$ H. H. Wicke and J. M. Worrell, Jr., Primitive structures in general topology, Studies in Topology, Academic Press, New York, 1975, pp. 581-599.

[ $\mathbf{W W}_{\mathbf{2}}$ ] Canad. J. Math. 27 (1975), 1100-1109.

Department of Mathematical Sciences, University of Texas at el Paso, El PASO, TEXAS 79968

Department of Mathematics, Ohio University, Athens, Ohio 45701 